Predation of the Noctilio albiventris (Chiroptera: Noctilionidae) by Pseudopimelodus schultzi (Siluriformes: Pseudopimelodidae) in the river basin La Vieja, Colombia

\title{
Depredación de Noctilio albiventris (Chiroptera: Noctilionidae) por Pseudopimelodus schultzi (Siluriformes: Pseudopimelodidae) en la cuenca del río La Vieja, Colombia
}

\section{Alvaro Botero-Botero ${ }^{1^{*}}$, Jairo Pérez-Torres ${ }^{2}$, Jhon Anderson Arcila-Marulanda ${ }^{3}$ y Julián Andrés Sánchez-Pachón ${ }^{4}$}

\begin{abstract}
'Grupo de investigación en Biodiversidad y Educación Ambiental: BIOEDUQ. Universidad del Quindío. Colombia. E-mail:abotero@ uniquindio.edu.co (AB-B).

${ }^{2}$ Laboratorio de Ecología Funcional, Unidad de Ecología y Sistemática (UNESIS), Departamento de Biología, Facultad de Ciencias, Pontificia Universidad Javeriana. Carrera 7 No. 40 - 62. Bogotá, D. C., Colombia. E-mail: jairoperez@javeriana.edu.co (JP-T).

${ }^{3}$ Grupo de investigación Biodiversidad y Educación Ambiental - BIOEDUQ, Universidad del Quindío. Av. Bolívar, carrera 15 calle 12 Norte. Armenia, Colombia. E-mail: jhonan@gmail.com (JAA-M).

${ }^{4}$ Programa de Lic. en Biología y Educación Ambiental, Universidad del Quindío. Av. Bolívar, carrera 15 calle 12 Norte. Armenia, Colombia. E-mail: julian.jaguar@gmail.com (JAS-P).
\end{abstract}

Introduction: Trophic interactions between fishermen and fish bats are common and most of the times the bat is the predator and fish, the prey. However, so far they have not been registered opposite events.

Methods: During a sampling sesion of fish in the the river basin of La Vieja, high Cauca (Colombia) in 2009, a toad catfish Pseudopimelodus schultzi was captured and after stomach analysis, an individual of the bat Noctilio leporinus was found.

Results and discussion: We report the first event of predation of $N$. albiventris by $P$. schultzi. This finding may have three possible explanations: 1) Opportunistic predation. 2) Active predation or 3) Opportunistic consumption.

Keywords: greater bulldog bat; Schultz's Bumblebee Catfish; upper Cauca basin.

Introducción: Son frecuentes las interacciones tróficas entre murciélagos pescadores y peces. En la mayoría de los casos, el murciélago es el depredador y el pez la presa. Hasta ahora no se han registrado eventos contrarios.

Métodos: En un muestreo de peces en la cuenca del río La Vieja, alto Cauca (Colombia), el 16 y 17 de mayo de 2009, fue capturado un bagre sapo Pseudopimelodus schultzi. Después de su procesamiento, se encontró un murciélago dentro de su estómago.

Resultados y discusión: Reportamos el primer evento de depredación de N. albiventris por P. schultzi. Este evento puede tener tres posibles explicaciones: 1) Depredación oportunista. 2) Depredación activa o 3) Consumo oportunista.

Palabras claves: alto Cauca; bagre sapo; murciélago pescador. 


\section{Introducción}

Son frecuentes las interacciones tróficas entre los murciélagos pescadores del género Noctilio y los peces. En todos los casos, el murciélago es el depredador y los peces las presas, como lo han mostrado diversos estudios (Bloedel 1955; Altenbach 1989; Brooke 1994; Aihartza et al. 2003; Bordignon 2006; Bordignon y França 2014). Sin embargo, hasta ahora no se han registrado casos en que el pez deprede al murciélago.

Los murciélagos de la Familia Noctilionidae están ligados a los ecosistemas acuáticos, debido a sus hábitos alimentarios. Estos murciélagos realizan vuelos rasantes sobre la superficie del agua (Tirira 2007). Noctilio albiventris es un murciélago que se alimentan de insectos ligados al ecosistema acuático, como los escarabajos, las chinches, moscas, libélulas, efemerópteros y mariposas nocturnas. También puede llegar a capturar peces en pozas con profundidad menor a $50 \mathrm{~cm}$ (Tirira 2007). Las especies de este género presentan una morfología adecuada para capturar presas sobre la superficie del agua (Kalko et al. 1998). Se ha considerado a esta especie como un frugívoro ocasional (Gonçalves et al. 2007), y por lo tanto, un potencial dispersor de semillas en áreas perturbadas cerca de bosques secos (Aranguren et al. 2011).

El pez sapo Pseudopimelodus schultzi es en Colombia una especie casi amenazada (Ortega-Lara et al. 2011; Ortega-Lara 2012). Esta especie es un pez carnívoro que se alimenta principalmente de peces, larvas de insectos acuáticos y detritus (Maldonado-Ocampo et al. 2005). También lo consideran de hábitos omnívoros porque se alimenta de peces como Astyanax fasciatus, Leporinus striatus, Pimelodus sp., Heptaptéridos y restos de materia vegetal (Ortega-Lara et al. 2011). Hábitos alimenticios similares se han reportado en P. bufonius (= Pseudopimelodus zungaro), en la cuenca del río La Vieja (Alto Cauca, Colombia), donde depredan otros peces como Imparfinis nemacheir y Cetopsorhamdia boquillae. Se han registrado restos de vértebras, escamas y aletas, y material de origen vegetal, como Guadua angustifolia (Román-Valencia 2004). Este pez presenta hábitos bentónicos y se oculta durante el día entre la vegetación sumergida, restos vegetales y rocas, especialmente en las zonas profundas de los grandes ríos. Prefiere los sustratos rocosos o con gravas, donde la corriente va de fuerte a moderada (Maldonado-Ocampo et al. 2005).

Un ejemplar de bagre sapo Pseudopimelodus schultzi se capturó entre el 16 y 17 de mayo de 2009. El posterior análisis estomacal de este individuo mostró un Noctilio albiventris en el estómago. Es de resaltar que el único contenido que se encontró en el estómago del bagre sapo fue el murciélago.

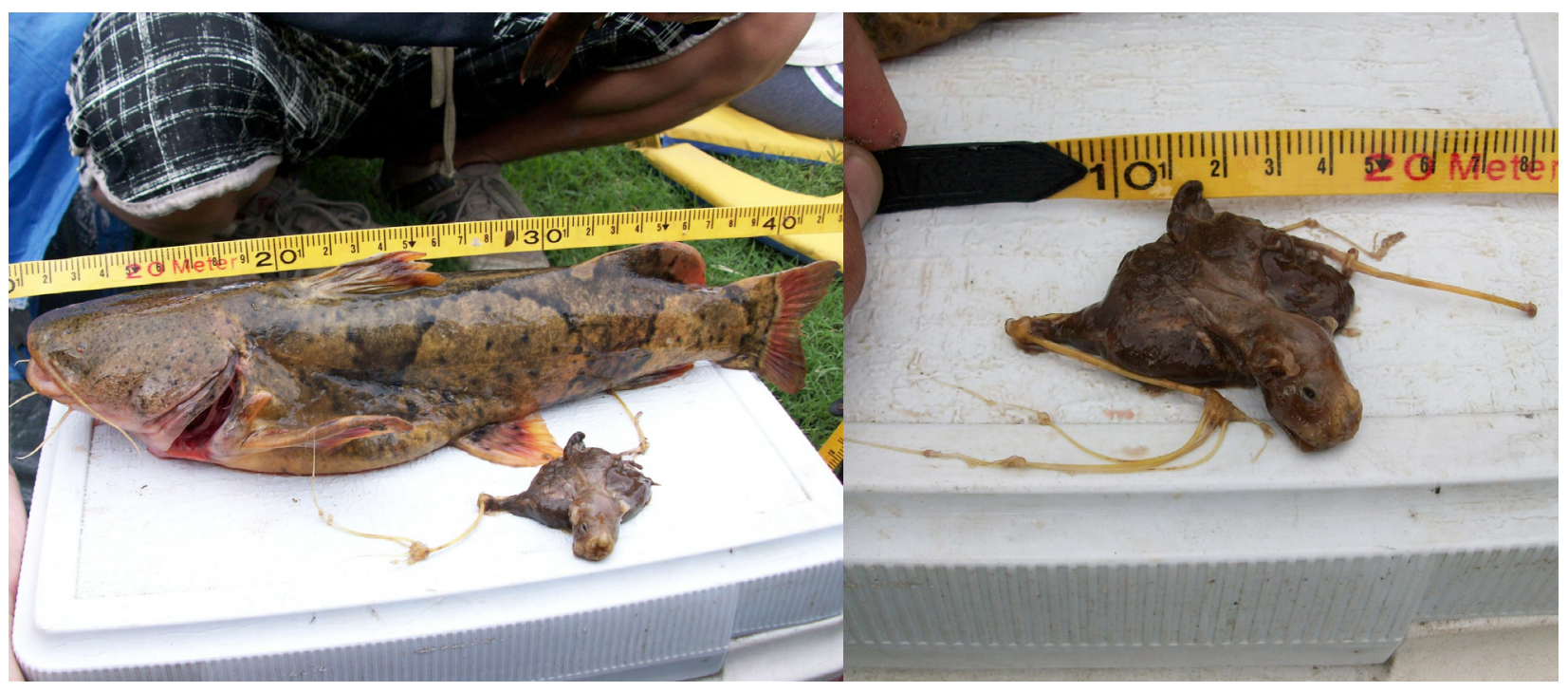

Figura 1. Registro fotográfico del bagre sapo (Pseudopimelodus schultzi) y el murciélago pescador (Noctilio albiventris). 
Noctilio albiventris presentaba un avanzado estado de descomposición. Se pudieron determinar las siguientes medidas: longitud del antebrazo de $45.0 \mathrm{~mm}$; longitud del cuerpo de 57.8; envergadura de $380.0 \mathrm{~mm}$; longitud de la oreja de $12.3 \mathrm{~mm}$. No fue posible identificar el sexo, ya que la parte inferior del cuerpo estaba descompuesta. Por comparación de bases de datos de la colección de mamíferos del Museo Javeriano de Historia Natural, se concluyó que las medidas corresponden a un individuo en etapa entre juvenil y subadulto. A su vez, la especie es común en la cuenca (Castaño-Salazar y Botero-Echeverry 2004).

El sitio de captura fue la cuenca media del río La Vieja, sector de Peña Santa, municipio La Tebaida, departamento del Quindío (4 $27^{\prime} 19^{\prime \prime} \mathrm{N}$ y $75^{\circ} 52^{\prime} 06^{\prime \prime} \mathrm{W}$ ) a 1,014 m. La precipitación promedio anual es de 1,100 mm/año, y la temperatura promedio anual, de $24^{\circ} \mathrm{C}$ (CRQ 2005). La vegetación circundante corresponde a Bosque muy húmedo Montano bajo (bmh-MB) (Asociación Red Colombiana de Reservas Naturales de la Sociedad Civil 2006), en la cual predomina una matriz de paisaje dominado por cultivos y pasturas para la ganadería. Las orillas del río están dominadas por bosques mixtos de guaduas y algunos árboles grandes.

Respecto de la observación de consumo de N. albiventris por parte del bagre sapo, pueden plantearse tres posibles explicaciones: 1) Depredación oportunista: el murciélago, al estar forrajeando, cayó accidentalmente al agua, y mientras trataba de salir, el bagre aprovechó la oportunidad para consumirlo. 2) Depredación activa: el murciélago se encontraba perchado en la saliente de alguna una roca emergente del río o en alguna rama muy cerca a la superficie del agua y el bagre lo depredó activamente, y 3) Consumo oportunista: el murciélago estaba muerto en el río y el bagre, durante su búsqueda cotidiana de alimento, lo encontró y consumió.

\section{Literatura citada}

Aihartza, J. R., U. Goiti, D. Almenar, y I. Garín. 2003. Evidences of piscivory by Myotis capaccinii (Bonaparte, 1837) in southern Iberian Peninsula. Acta Chiropterologica 5:193-198.

Altenbach, J. S. 1989. Prey capture by the fishing bats Noctilio leporinus and Myotis vivesi. Journal of Mammalogy 70:421-424.

Aranguren, C. I., J. A. González-Carcacía, H. Martínez, y J. M. Nassar. 2011. Noctilio albiventris (Noctilionidae), a Potential Seed Disperser in Disturbed Tropical Dry Forest Habitats. Acta Chiropterologica 13:189-194.

Asociación Red Colombiana de Reservas Naturales de la Sociedad Civil. 2006. Estrategia de conservación regional - nodo Eje Cafetero. Proyecto promoción y consolidación de reservas naturales de la sociedad civil en los andes colombianos. Convenio Instituto Humboldt - Red. Armenia, Colombia.

Bloedel, P. 1955. Hunting methods of fishing-eating bat, particulary Noctilio leporinus. Journal of Mammology 36:390:399.

Bordignon, M. O. 2006. Diet of the fishing bat Noctilio leporinus (Linnaeus) (Mammalia, Chiroptera) in a mangrove area of southern Brazil. Revista Brasileña de Zoologia 23:256-260.

Bordignon, M. O., y A. O. França. 2014. First consumption by Noctilio leporinus (Linnaeus, 1758) in Guaratuba Bay, Southern Brazil. Chiroptera Neotropical 8:148-150.

Brooke, A. P. 1994. Diet of the fishing bat Noctilio leporinus (Chiroptera: Noctilionidae). Journal of Mammalogy 75:212-218.

Castaño-Salazar, J. H., y J. E. Botero-Echeverry. 2004. Murciélagos de la zona cafetera colombiana. Avances Técnicos CENICAFE 329:1-8.

CRQ (Corporación Autónoma Regional del Quindio). 2005. Plan de Ordenamiento y Manejo de la Cuenca del río La Vieja, diagnóstico julio de 2005. (Acceso agosto 15 de 2010) URL http://www.crq.gov.co/ visual_crq/documentos/proyecto_riolavieja/diagnostico_rio_lavieja.pdf.

Gonçalves F., R. Munin, P. Costa, y E. Fischer. 2007. Feeding habits of Noctilio albiventris (Noctilionidae) bats in the Pantanal, Brazil. Acta Chiropterologica 9:535-546. 
Kalko, E., S. Hans-Ulrich, I. Kaipf, y A. D. Grinnell. 1998. Echolocation and foraging behavior of the lesser bulldog bat, Noctilio albiventris: preadaptations for piscivory? Behavioral Ecology and Sociobiology 42:305-319.

Maldonado-Ocampo, J. A., A. Ortega-lara, J. S. Usma, G. Galvis, F. A. Villa-Navarro, G. L., S. Vásquez, Y. Prada-Pedreros, y R. C. Ardila. 2005. Peces de los Andes de Colombia. Instituto de Investigación de Recursos Biológicos Alexander von Humboldt. Bogotá, Colombia.

Ortega-Lara, A. 2012. Pseudopimelodus schultzi. Pp. 246-248 en Libro rojo de peces dulceacuícolas de Colombia (Mojica, J. I., J. S. Usma, R. Álvarez-León, y C. A. Lasso Eds.). Instituto de Investigación de Recursos Biológicos Alexander von Humboldt, Instituto de Ciencias Naturales de la Universidad Nacional de Colombia, WWF Colombia y Universidad de Manizales. Bogotá, Colombia.

Ortega-Lara, A., T. S. Rivas-Lara, y C. E. Rincón. 2011. Pseudopimelodus schultzi (Siluriforme, Pseudopimelodidae). Pp. 547-550 en I Catálogo de los recursos pesqueros continentales de Colombia (Lasso, C., E. Agudelo-Córdoba, L. Jiménez-Segura, H. Ramírez-Gil, M. Morales Betancourt, R. E. Ajiaco-Martínez, E. A. Muñoz-Torres, y I. Sanabria-Ochoa Eds.). Instituto de Investigaciones de Recursos Biológicos Alexander von Humbolt. (IAvH). Bogotá, Colombia.

Román-Valencia, C. 2004. Datos Bioecológicos del peje sapo, Pseudopimelodus zungaro (Pisces: Pimelodidae), de los ríos Atrato y La Vieja, Colombia. Dahlia Revista de la Asociación Colombiana de Ictiologia 7:29-31

TiriRA, D. 2007. Mamíferos del Ecuador. Guía de campo. Ediciones Murciélago Blanco. Quito, Ecuador.

Summited: April 13, 2015

Review: May 29, 2015

Accepted: August 18, 2015

Associated editor: Cristina Macswiney 